\title{
Definition of a responder in clinical trials for functional gastrointestinal disorders: report on a symposium
}

\section{Introduction}

On 10 and 11 September 1998, about 125 participants met at a symposium in Vienna, Austria, with the goal of determining whether a consensus could be developed on the definition of responders in clinical trials in the functional gastrointestinal disorders. Present at this meeting were representatives of the Food and Drug Administration (FDA) (Robert Prizont, MD, and John Senior, $\mathrm{MD}^{\star}$ ), a representative of the Japanese International Motility Society (Kei Matsueda, MD, PhD), academic investigators from around the world, and representatives of several pharmaceutical companies currently engaged in the development of therapeutic compounds for the functional gastrointestinal disorders. The discussion centered primarily on the irritable bowel syndrome (IBS), the functional gastrointestinal disorder for which there is the largest development program underway. However, the discussion also addressed the measurement of outcomes in functional constipation.

This conference was sponsored by the Multinational Working Teams to Develop Diagnostic Criteria for Functional Gastrointestinal Disorders (MWT). Over a two year period, the executive committee of the MWT held a series of informal meetings with representatives of the FDA, and on one occasion, with a representative of the European Agency for the Evaluation of Medicinal Products. During these meetings, representatives of the regulatory groups indicated that there was a need for a more broad-based consensus on the best ways of defining responders in clinical trials. The MWT organized this conference to meet this need.

This conference was unique in several respects. It is the first time representatives of the regulatory agencies, the academic community, and the pharmaceutical research teams have met together with the goal of developing a consensus on outcome measurement. It also represents a milestone in the willingness of different pharmaceutical companies to share data from actual clinical trials for the purpose of developing outcome measures that could be used by all of them. The degree of consensus achieved at this initial meeting was modest. However, this represents the first step in a process that is expected to lead to a broad-based consensus on these issues.

The conference was organized as a multi-stage process. Firstly, representatives of the FDA and of the Japanese International Motility Society presented position papers; Dr Drossman described the history of the MWT; and Dr Sander Van Zanten presented the provisional recommendations of the MWT which dealt with the design of clinical trials. Following this, academic investigators described their views on the measurement of outcomes. The representatives of six pharmaceutical companies then presented data. These representatives were asked to select clinical trials completed by their companies in which at least one outcome measure had been found to be significantly different between drug and placebo groups, and to compare the different outcome measures used in these trials on a common scale, making it possible to compare their relative efficacy and the magnitude of the placebo response. These presentations were followed by an

A detailed summary of the conference is available on request.

${ }^{\star}$ Drs Senior and Prizont participated as individual scientists. Their views do not represent the official policy of the FDA. open discussion whose purpose was to assess the degree of consensus among the participants and to summarize their recommendations for measurement. Following the conference, these recommendations, together with a summary of the presentations at the conference, were circulated to the participants and to other members of the scientific community for comment. After this review process was completed, the recommendations were forwarded to the regulatory agencies and to the companies that participated in the conference.

\section{Consensus recommendations}

The participants were not able to reach a consensus on any single approach to the measurement of outcomes in clinical trials. However, the following points of agreement were reached:

- Evaluation should be based on patient reports rather than ratings by investigators/clinicians.

- Efficacy evaluation should be based on the percentage of subjects meeting a predefined criterion of clinical significance rather than on the statistical significance of differences between groups or between periods of observation.

- Outcome measures based on self-reported relief of symptoms seem to be promising because they are easily understood by patients and have face validity. However, such measures often have dichotomous response scales (e.g., yes or no), and there is evidence that dichotomous scales are associated with lower statistical power (greater error of measurement) than continuous measures or ordinal-scaled measures with more than five steps.

- Global ratings of symptom severity or symptom change in which the patient is asked to integrate his/her experience, or summated indexes such as validated questionnaires, seem to be as good as specific symptom measures.

- Retrospective ratings of "usual" symptom severity are generally good approximations of daily diary averages over brief periods such as one month or less.

- Visual analog scales (VAS) and numerical scales have been criticized because (a) about $10 \%$ of patients find them difficult to comprehend, and $(b)$ it has been difficult to define clinically significant change or group differences. However, simultaneous use of VAS or numerical rating scales of intensity, change, or relief can greatly complement and help in the interpretation of dichotomous or categorically scaled primary outcome measures. When VAS and numerical scales are used, patients should be carefully instructed.

- Quality of life scales, symptom severity indexes, and psychological or cognitive measures cannot be recommended as primary outcome measures at this time because they have not been sufficiently validated in the context of drug evaluation.

- Placebo run-ins are not recommended because they may interfere with the assessment of treatment effects, but baseline periods of measurement without treatment are acceptable.

- Psychological measures are recommended at baseline because they may be useful as covariates in the interpretation of differential treatment responses.

- Physiological investigations are encouraged because they advance our understanding of the disorder and the mechanism of therapeutic benefit, but they are not recommended for primary outcome measures. 
- Multinational treatment trials require attention to linguistic and cultural translation of outcome measures, and they require full disclosure of the methods used to develop and validate transcultural instruments.

- The cognitive aspects of outcome assessment, including the ability of patients to understand the questions and to make adequate numbers of discriminations, should be addressed in selecting outcome measures.

Supported by the UNC Center for GI Functional and Motility Disorders, the Multinational Working Teams to Develop Diagnostic Criteria for the Functional Gastrointestinal Disorders (Rome II), and by the following pharmaceutical companies: Astra-Zeneca, GlaxoWellcome, Janssen Pharmaceutica, Novartis, Pfizer, Roberts, SmithKline Beecham, Solvay Pharmaceuticals, and TAP Holdings.

\section{Chairperson}

W E WHITEHEAD

Professor of Medicine,

University of North Carolina at Chapel Hill,

North Carolina, USA

\section{Organizing Committee}

E CORAZZIARI

University of Rome,

Rome, Italy

R PRIZONT

J R SENIOR

Division of Gastrointestinal and Coagulation Drug Products,

Food and Drug Administration,

Rockville, Maryland, USA

W G THOMPSON

University of Ottawa,

Ottowa, Canada

S J O VELDHUYZEN VAN ZANTEN

Dalhousie University,

Halifax, Nova Scotia, Canada

Correspondence to: William E Whitehead, $\mathrm{PhD}$, Division of Digestive Diseases, CB \# 7080, Burnett-Womack Building, University of North Carolina at Chapel Hill, Chapel Hill, NC 27599-7080, USA. Email:

william_whitehead@med.unc.edu

\section{For further information and updates on Rome II, visit our website at: www.romecriteria.org}

